\title{
PATTERN-MATCHING COMMANDS
}

\author{
Jan B. Hext \\ Basser Department of Computer Science \\ University of Sydney \\ ABSTRACT
}

\begin{abstract}
The paper investigates the following question: what happens if we try to embed the pattern-matching facilities of snobol4 in a more conventional language? It shows that the facilities can be improved in some significant ways. However, the control structures that underlie pattern-matching operations are radically different from the usual ones, and this creates difficulties if the full power of the snobol 4 system is to be retained.
\end{abstract}

\section{SNOBOL4}

Snobol4 is well known as a significant and useful programming language and is widely taught for its ability to handle non-numeric computations (Griswold et al., 1971). Indeed, for some applications it makes most other languages appear crude and primitive. At the same time, it must be admitted that Snobol4 itself is open to criticism in some respects. For example, its notation is rather obscure, its control facilities are very limited, and its data structures have certain unorthodox aspects which constitute a definite health hazard. It is therefore reasonable to ask whether the best features of the language might be transferred to some other environment, where they could shine to better advantage. Our purpose in this paper is to investigate the factors which would be involved in such an enterprise. Some of the proposals have been implemented using BCPL as the host language; but any other generalpurpose language could be considered for the purpose.

Although Snobol4 has many facilities, our only concern will be with its most distinctive and interesting feature, namely the patternmatching statement. This has the form

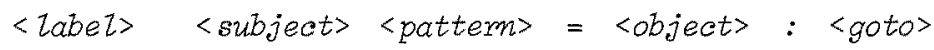

Its effect is to scan the string <subject> for an occurrence of the <patterns and, if the search is successful, to replace the matching substring by the string $\langle a b j e c t>$. The <goto> part consists of one or two clauses of the form (<zabez>), $\mathrm{S}(\langle z a b e Z\rangle)$ or $\mathrm{F}(\langle z a b e Z\rangle)$. These cause transfer of control to the specified labels, the last two being conditional on the success or failure of the scan. For example, the statement

$$
\text { L1 TEXT 'GREAT' = 'BIG': S(DONE) }
$$

will scan the value of TEXT for the first occurrence (if any) of the string 'GREAT' and will replace it by the string 'BIG'. If successful, 
it transfers control to the statement labelled DONE; otherwise it continues to the next statement.

The power of this facility lies in the great range of patterns which the programmer can specify and in the fact that various other operations can be executed during the pattern-matching process. The following are some simple examples of the available patterns:

\begin{tabular}{|c|c|c|}
\hline NULL & $\because$ & the null string \\
\hline $\operatorname{LEN}(5)$ & & any string of length 5 \\
\hline$A N Y\left({ }^{\prime} A B C^{\prime}\right)$ & 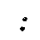 & any one of the letters $A, B, C$ \\
\hline $\operatorname{SPAN}(' A B C$ ') & $\because$ & $\begin{array}{l}\text { any sequence of the letters } A, B, C \\
\text { (such as } C A B \text {, or } A B B A \text {, or } C C C C C \text { ). }\end{array}$ \\
\hline
\end{tabular}

Strings and other basic patterns may be combined into pattern structures by means of alternation and catenation. Snobol4 denotes the former by ']' and the latter by actual concatenation (as in BNF). However, we shall use a more explicit notation:

$$
\begin{array}{ll}
P 1 \text { alt } P 2 \quad: \quad \text { any string that matches either } P 1 \text { or } P 2 \\
P 1 \text { cat P2 } & \text { any string that consists of a substring } \\
\text { that matches } P 1 \text { followed by a substring } \\
\text { that matches } P 2 .
\end{array}
$$

These are familiar as the first two operations of regular expressions. The third operation, namely repetition, is provided by the pattern $A R B N O(P)$, which matches nought, one or more occurrences of the pattern $P$. There are obvious counterparts in the basic control structures of programs, namely if-statements, sequencing and loops. However, as we shall see later, the analogy needs to be treated with caution.

There are two facilities for executing operations during the pattern-matching process. The first of these is value assignment, which allows a matching substring to be assigned to a variable. In the simplest case, a variable $V$ can be associated with a pattern $P$ in such a way that, whenever a search for $P$ is successful, the matching substring is immediately assigned to $V$. This applies even when $P$ is part of a larger pattern and ever if $P$ is matched several times during the complete operation.

The second facility is the use of "pseudo-patterns". These may be defined as patterns whose primary role is not to match particular strings but to produce specific side-effects. For example, the expression $a X$ is defined to be a pattern structure that matches the null string; but its real purpose is to assign to the variable $X$ the position of the cursor (the pointer that is used for the scanning operation). For example, if TEXT has the value 'THE GREAT AUSTRALIAN BYTE', the statement

\section{TEXT 'GREAT' QXX}

matches 'GREAR' followed by the null string. As a side-effect, it assigns $X$ the value 9 , indicating the position that was reached after 'GREAT'.

The pxogrammer can define his own functions and have them called as pseudo-patterns in a similar way. This enables him to execute arbitrary operations during the pattern-matching process. The use of 
pseudo-patterns is rather artificial, but the underlying facility adds greatly to the power of the language.

\section{A SCAN COMMAND}

If we embed the above pattern-matching command in some other language, we may prefer to make it more explicit:

$$
\text { <string variable> mateh <pattem> := <string expression> }
$$

The match operator may be understood as designating a substring of the variable; the substring (if found) is replaced by the value of the <string expression>. A flag may be set to indicate whether or not the matching was successful.

This transliteration is an obvious starting point. Unfortunately, though, it preserves one of the main deficiencies of the snoboll system. The trouble is that it does not allow for more than one match operation to be executed during a single scan of the string. To illustrate the resulting drawbacks, we may extend the GREAT-BIG substitution problem so that it requires every occurrence of 'GREAT' to be replaced by 'BIG'. The technique commonly used in the textbooks is to use the following loop:

$$
\text { LOOP TEXT 'GREAT' = 'BIG' : S(LOOP) }
$$

This certainly solves the problem. However, for each substitution that it makes, it requires (a) that the string be reconstructed and (b) that the next scan start afresh from the first character. If TEXT is more than a few lines long, this is clearly very inefficient. There are ways of speeding it up - e.g., by using the ' $a$ ' operator to record the current position - but none can be regarded as satisfactory.

To avoid this inefficiency, we can take a cue from conventional text editors. They operate on an input string $S 1$ and produce an output string S2, carrying out an arbitrary sequence of operations within a single scan. This suggests that the previous command be rewritten in the following form:

$$
\text { S2 }:=\quad \text { scan } S 1 \text { do <statement sequence> }
$$

Within the <statement sequence> we provide the ability to search for patterns starting from the current position of the scan. The GREAT-BIG substitution problem can then be programmed as follows:

$$
\begin{aligned}
& \text { text }:=\text { scan text do } \\
& \text { repeat } \\
& \text { match 'great' }:=\text { 'big' }
\end{aligned}
$$

The condition endstring is automatically set to true when the scanner reaches the end of the string. The resulting statement expresses what we want to do in an elegant and efficient way.

Within the scan command we could include many other special operations. For example, we could copy characters, skip characters, insert characters, and so on. Text editors provide a wealth of facilities 
which deserve consideration in this regard. However, the precise details would doubtless be a matter of some controversy and it is not our intention to discuss them here. Besides these special operations, though, the body of the scan may include all the ordinary statements of the language. Thus assignments, procedure calls, etc. may be intexspersed with patternmatching commands in an arbitrary fashion.

\section{PATTERNS WHICH ACTIVATE COMMANDS}

If we implement the above proposal, one of the deficiencies of Snobol4 will immediately disappear - namely, its inability to do several match operations in a single scan. On the face of it, we might expect another problem to disappear also - namely, the need for pseudo-patterns. The point is that we can insert ordinary statements between patternmatching commands; so why bother to insert them into the patterns themselves? All we need do is convert a pattern of the form $P 1$ alt $P 2$ into the two branches of an if-statement and a pattern of the form $P 1$ cat $P 2$ into two sequential statements; then, surely, we can insert side-effects quite freely.

Unfortunately, the conclusion does not follow quite so easily. The alt and cat operators cannot be equated with conventional control mechanisms in such a simple manner. The trouble is that a pattern such as

\section{(P1 azt P2) eat $P 3$}

has the following implication. If we succeed in matching P1 but then fail to match $P 3$, we must go back and try $P 2$ instead. In fact, if there are alternatives within $P 1$, we must try them also. This means that a pattern has the nature of a semi-coroutine: after matching it successfully, we must be able to resume it later in order to look for alternatives. In general, therefore, a pattern-matching statement cannot be transformed into a more primitive sequence of statements except by introducing some rather elaborate apparatus. As a result, it would be more convenient if some other method were provided for including arbitrary statements within the pattern-matching process.

From the above observations, we may conclude that the natural thing to do is to allow statements to be specified within the pattem itself. As we have seen, snobol 4 provides two facilities for this, namely value assignments and pattern functions. Both of these have a rather ad hoc flavour. As an alternative, we might therefore consider the possibility of allowing an arbitrary statement to be attached to a pattern or subpattern. Taking the notation from Snobol's 〈goto field, we can express the idea by means of the following pattern extensions:

$$
\begin{gathered}
\text { <pattem> }: \quad \text { (<statement sequences) } \\
\text { <patterm> } \\
\text { <pattem> }: \quad F \text { (<statement sequence>) }
\end{gathered}
$$

The first executes the <statement sequence> whenever a match against the <patterpl> is attempted. The other two execute it conditionally, depending on whether the match succeeds or fails. 
As an example, let us consider the problem of counting the number of vowels and consonants in the string text. Using the above facility, we can proceed as follows:

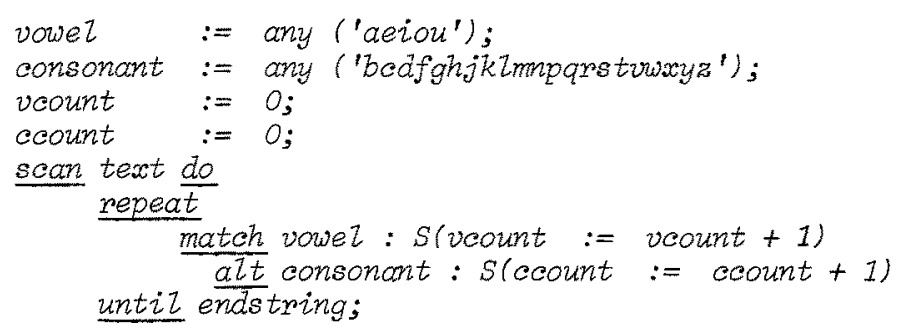

This is much neater than matching vowel alt consonont and then having to determine which of the two (if either) was successful. The facility also provides a natural context for doing value assignment. We could introduce a quantity pat (say) that represents the matching substring; it can then be assigned to a variable using an ordinary assignment statement.

\section{PATTERN-MATCHING PROCEDURES}

If we adopt the above policy, it is clear that patterns can no longer be regarded simply as templates for matching strings. Instead, they have developed into a special sort of procedure. The procedure is characterized by a control structure which is based on the alt and cat operators and which executes related statements according to the progress of the scan. Snobol4 recognizes the special nature of this control structure by introducing four pseudo-patterns which can further alter its course - namely, ABORT, FENCE, SUCCEED and FAIL. We might therefore incorporate these as special directives within our new facility. They would have a status similar to the exit, $200 p$ and retum commands which are sometimes found in ordinary programing languages.

In order to regulate the use of such facilities, there are advantages in introducing explicit pattem procedures. Their declaration might take a form similar to that of other procedures:

$$
\text { pattem } P \text { (<parometer list }) ; \ldots
$$

The use of the special control facilities could then be restricted to this context. Instances of $P$ could be generated in the context of the match command or as procedure parameters. Like other functions and procedures, though, they would not normally be assignable to variables.

As in Snobol4, the use of pattern procedures enables patterns to be defined recursively. This has the attraction that it opens up the whole area of syntax analysis, with each category of the syntax being represented by a separate pattern procedure. The prospect is appealing. However, it should be noted that the pattern-matching algorithm could prove rather slow for the purpose and it would run into difficulties with left-recursive categories. 


\section{CONCLUSION}

The above proposals embody two basic pxinciples for the inclusion of pattern-matching commands within the framework of a general-purpose language. They show that the provision of a useful string-processing facility is not simply a matter of adding a few operators. Indeed, the difficulty of the enterprise may explain why conventional languages pay such scant attention to this axea of need.

It will be appreciated, of course, that neither of the two principles is at all original. As already remarked, the sequential scanning operation is Eamiliar enough in text editing and it has an earlier ancestry in the updating of sequential files. The concept of associating statements with patterns goes back to the first syntax-directed compilexs (Irons, 1961). More recently, it has appeared in AI languages such as QIisp, Planner and Conniver, where it is known as "the patterndirected invocation of commands" (McDermott and Sussman, 1972).

As far as the implementation is concerned, it will be clear that strings must be given the type sequence of char rather than array of char. A recent report by A.H.J. Sale (1978) argues the case for doing this in the context of Pascal and makes specific recomendations for the necessary language changes.

Space precluaes a presentation of the basic algorithm for implementing the pattern-matching comand - that is, for matching a string against a pattern with alt and cat operators. Since no easy-to-follow description appears to have been published in the literature, the author has prepared an elaboration of the outzine given by Harrison (1973) and would be happy to supply copies of it on request. Griswold (1977) discusses language facilities for implementing such algorithms at a higher level.

\section{REFERENCES}

Griswold, R.E. (1977) : "Language facilities for programmable backtracking", Proceedings of the Symposium on Artificial Intelligence and Programing Languages, SIGPLAN Notices, Vol.12, No.8, and SIGART Newsletter, No. 64 .

Griswold, R.E., Poage, J.F. and Polonsky, I.E. (1971): The Snobol4 Programing tanguage, 2nd edition, Prentice-Hall, Englewood Cliffs, NJ.

Harrison, M.C. (1973) : Data Stmuctures and Programming, Scott Foresman and Co., Glenview, Illinois.

Irons, E.T. (1961) : "A syntax directed compiler for Algol 60", Comm. ACM, Vol.4, po. 51-55.

McDermott, D.V. and Sussman, G.J. (1972): "From planner to Conniver - a genetic approach", Proc. FJCC, 1171-11.79.

Sale, A.H.I. (1978) " "Strings and the sequence abstraction in PascaI", Report R78-4, Department of Information Science, University of Tasmania. 